\title{
Development of bimetal oxide doped multifunctional polymer nanocomposite for water treatment
}

\author{
Swati Saxena ${ }^{1,3} \cdot$ Umesh Saxena ${ }^{2,4}$
}

Received: 9 January 2016/ Accepted: 16 August 2016/Published online: 29 August 2016

(c) The Author(s) 2016. This article is published with open access at Springerlink.com

\begin{abstract}
Bimetal oxide doped polymer nanocomposite was developed using Alumina and Iron (III) Oxide as nanoparticles with Nylon 6, 6 and Poly (sodium-4-styrenesulphonate) as polymer matrix for removal of pollutants from water. The blend sample of polymers was prepared by well established solution blending technique and their nanocomposite samples were prepared through dispersion technique during the solution casting of blend sample. The fabricated composites were characterized adopting FTIR, XRD, FESEM and EDX techniques. XRD and FESEM were used for morphological characterization of nano phase, while FTIR and EDX analysis were adopted for characterization of chemical moieties in composites. In the study of pollutant removal capacities of prepared composites, $6 \%$ nanocomposite provided the best results. It exhibited the maximum removal of all parameters. The removal of total alkalinity was $66.67 \%$, total hardness $42.85 \%$, calcium $66.67 \%$, magnesium $25 \%$, chloride $58.66 \%$, nitrate $34.78 \%$, fluoride $63.85 \%$, TDS $41.27 \%$ and EC was up to the level of $41.37 \%$ by this composite. The study is a step towards developing multifunctional,
\end{abstract}

Umesh Saxena

saxenaumesh@yahoo.com

Swati Saxena

swati.snigdha11@gmail.com

1 Department of Chemistry, Suresh Gyan Vihar University, Jaipur, Rajasthan, India

2 U. S. Ostwal College of Science, Chittorgarh, Rajasthan, India

3 Present Address: 53, Vrindavan Colony, Near Khatipura Over Bridge, Jhotwara, Jaipur, Rajasthan 302012, India

4 Present Address: F-202, Shyam Nagar Ext. Ram Path, Sodala, Jaipur, Rajasthan 302019, India cost-effective polymer nanocomposites for water remediation applications.

Keywords Nanocomposite - Water treatment - Alumina . Iron (III) oxide $\cdot$ Nylon $6,6 \cdot$ Poly

(sodium-4-styrensulphonate) $\cdot$ Pollutants

$\begin{array}{ll}\text { Abbreviations } \\ \text { DM } & \text { Demineralised } \\ \text { EDX } & \text { Energy dispersive X-ray analysis } \\ \text { FESEM } & \text { Field emission scanning electron microscopy } \\ \text { FTIR } & \text { Fourier transform infrared } \\ \text { MMM } & \text { Mixed matrix membrane } \\ \text { MWCNTs } & \text { Multi-walled carbon nano tubes } \\ \text { PEG } & \text { Poly ethylene glycol } \\ \text { PSS } & \text { Poly (sodium-4-styrenesulphonate) } \\ \text { SEM } & \text { Scanning electron microscopy } \\ \text { TDS } & \text { Total dissolved solids } \\ \text { XRD } & \text { X-ray diffraction }\end{array}$

\section{Introduction}

At present, water shortage and poor water quality are the major crises in many parts of the world. According to the World Health Organization, 1.1 billion people lack access to improved drinking water and 2.6 billion have no access to proper sanitation [1]. As many as 2.2 million people die of diarrhea related diseases every year most often caused by water borne infections, and the majority of these cases are children under the age of 5 [2].

Most of the surface and ground water sources in India are getting increasingly polluted due to onslaught of human activities. India is heading towards a freshwater crisis mainly due to improper management of water resources, 
and environmental crisis is already evident in many parts, varying in scale and intensity [3]. Only $12 \%$ of the people get clean drinking water, and the rest quench their thirst with polluted lakes, tanks, rivers and wells due to which more than three million people are affected or die of enteric diseases every year [4]. The existing fresh water resources need protection more than ever, and new water resources must be developed to meet the growing demand of potable water which requires better water treatment technology.

Nanotechnology has been identified as an important tool to address the environmental problems like health and medicine, air, water pollution, etc. Over the past decade, nanotechnology has rapidly changed from an academic pursuit to a commercial reality; already nanotechnology concepts have led to new water treatment membranes that exceed state-of-the-art performance. Due to their ease of operation and greater efficiency, nanofibrous membranes will play an important role in the replacement of conventional membranes in the near future [5]. Incorporation of nanotechnology in membrane preparation could offer an attractive alternative to prepare real fouling and other pollutant resistant membranes and hence many scientists believe that nanotechnology would bring revolutionary advances to the desalination industry.

Recently, iron oxide based nanocomposite was developed and found to have high fluoride removal capacity $(\sim 97 \%)$ at a contact time of $60 \mathrm{~min}$ [6]. The nano-MgO on PEG (poly ethylene glycol) exhibited the largest adsorption capacity of $74 \mathrm{mg} / \mathrm{g}$ for fluoride [7]. Tomar and Kumar reviewed the efficiency of different materials for fluoride removal from aqueous media and stated that nano adsorbents have attracted considerable attention in the recent years in fluoride removal and these materials have shown higher fluoride uptake capacity [8]. Granular ferric hydroxide and nano $\mathrm{Al}_{2} \mathrm{O}_{3}$ were also tested for the removal of fluoride, perchlorate and nitrate anions from aqueous solutions [9]. A bimetal doped ( $\mathrm{Al} \& \mathrm{Fe}$ ) micro- and nano multifunctional polymeric adsorbents were prepared for the removal of fluoride and arsenic (V) from wastewater [10]. Fluoride removal from water solution by adsorption on activated alumina prepared from pseudo-boehmite was also investigated and it was depicted that fluoride adsorption capacity of the activated alumina considerably depends on the solution $\mathrm{pH}$ and diminishes with increasing solution $\mathrm{pH}$ from 4 to 11 [11].

Manimegalai et al. reviewed the pesticide degradation by supported silver nanoparticles and proved that silver nanoparticles have the ability to mineralize pesticides but supporting materials for these particles are yet to be explored. According to them, polyurethane foam coated with silver nanoparticles could be a promising option as supporting media [12]. With respect to the above research,
Manimegalai et al. synthesized cellulose acetate membrane incorporated with silver nanoparticles to effectively study the mineralization of pesticides and found that silver nanoparticles do not discriminate among different pesticides; and that the concentration of nanoparticles enhances the rate of mineralization. It can be effectively used for the removal of pesticides in rural areas where the pesticide contamination is prevalent [13]. Chitosan-silver nanopaticle composite was also prepared and analyzed for its pesticide removal capacity and demonstrated that microbeads of the composite are excellent agents for the removal of Atrazine from aqueous solution at neutral $\mathrm{pH}$ under equilibrium and column flow experimental conditions [14].

Issues of silver nanoparticles in engineered environmental treatment systems were studied and it was found that these particles may significantly influence chemical and biological processes in these treatment systems [15].

Nitrate removal was studied by clinoptilolite zeolite. Totally, it did not exhibit sufficient efficiency for nitrate removal [16]. Ahmad et al. concluded that alumina supported nano zero valent zinc as adsorbent showed better efficiency for removal of arsenic and nitrate ions [17]. Mixed matrix membrane (MMM) of polyacrylonitrile and alumina nanoparticles also showed the adsorptive removal of nitrate from aqueous solution [18]. The system $\mathrm{Fe}_{3} \mathrm{O}_{4} /$ Poly Aniline was successfully tested for a high removal efficiency of nitrate from urban wastewater [19]. Nabid et al. studied the removal of toxic nitrate ions from drinking water using polymers/MWCNTs nanocomposite conduction. According to them, the highest removal of nitrate was achieved by Polyaniline/MWCNTs nanocomposite [20]. Bhatnagar et al. conducted their study to evaluate the feasibility of nano alumina for nitrate removal from aqueous solutions. Their findings showed that the maximum sorption capacity of nano alumina for nitrate removal was found to be $4.0 \mathrm{mg} \mathrm{g}^{-1}$ at $25 \pm 2{ }^{\circ} \mathrm{C}$ [21].

Sedaghat and Nasseri synthesized and stabilized the silver nanoparticles on a polyamide (nylon 6,6) surface and studied its antibacterial effects [22]. Ion exchanger nanofibrous polystyrene matrix was prepared and found to have rapidly faster kinetics of the sorption properties in comparison to the granular ion exchangers [23].

Review of the literature shows that even though many works have been carried out with polymer metal nanocomposites, very few literatures are available on alumina and iron oxide combined loaded polymer nanocomposites and if at all prepared they are mainly focused on removal of any one or two particular principal pollutants from water. Hence, in this study an attempt is made to prepare multifunctional, cost effective polymer metal nanocomposite using alumina and iron oxide as nanoparticles with Nylon 6, 6 and Poly (sodium-4-styrenesulphonate) (PSS) as polymer matrix to reduce various pollutants from water. 


\section{Experimental}

\section{Chemicals}

All chemicals were procured from Sigma-Aldrich, US and were of analytical research grade. Nylon 6, 6 (in pellet form) and Poly (sodium-4-styrenesulphonate) (solution form) were used for the blend preparation in the present work. Alumina (alpha) nanopowder and Iron (III) oxide nanopowder were used for nanocomposite preparation and formic acid was used as solvent.

\section{Synthesis of Nylon/Sulphonate blend sample (polymer composite)}

The blend sample of nylon $\left(\mathrm{P}_{1}\right)$ with Poly (sodium-4styrenesulphonate) $\left(\mathrm{P}_{2}\right)$ was prepared in compositions of $1: 1$ by weight through solution blending technique. $1 \mathrm{~g}$ nylon was dissolved in $50 \mathrm{~mL}$ of formic acid at room temperature with continuous stirring through magnetic stirrer for about an hour. After, the formation of a clear solution; poly (sodium-4-styrenesulphonate) solution was mixed in the nylon solution. The resulting solution was again continuously stirred and then poured into flat bottomed glass petri dish. The solvent was allowed to evaporate slowly over a period of $48 \mathrm{~h}$ in dry atmosphere and next $24 \mathrm{~h}$ in vacuum dessicator to ensure the removal of the residual solvent. Then, it is kept in DM (demineralised) water for $24 \mathrm{~h}$ for the removal of strains of formic acid, if there is any and again dried $24 \mathrm{~h}$ in atmosphere and $24 \mathrm{~h}$ in vacuum dessicator. The composite so obtained was then separated for further characterization and studies.

\section{Synthesis of Alumina and Iron (III) oxide nanocomposite samples of Nylon/PSS polymeric blend}

The alumina $\left(\mathrm{N}_{1}\right)$ and Iron (III) oxide $\left(\mathrm{N}_{2}\right)$ nanocomposite samples of Nylon/Poly sulphonate were prepared by dispersing alumina and Iron (III) Oxide nanoparticles during the solution casting of polymeric blend. In this method, nylon was first dissolved in formic acid and then mixed with poly sulphonate solution as mentioned above. Then, 2, 4, 6 and $8 \%$ alumina and Iron (III) oxide nanoparticles (1:1) by weight of blending composition were dispersed in the solutions. The nanoparticles were dispersed by gentle stirring for about $2 \mathrm{~h}$ to ensure the uniform distribution of nanoparticles in polymeric blend solutions and then poured into flat bottomed glass petri dishes. The composites were then subjected to dry atmosphere for $48 \mathrm{~h}$ and vacuum dessicator for $24 \mathrm{~h}$ accordingly for the removal of solvent; then, these are kept in DM water for
$24 \mathrm{~h}$ for the removal of strains of formic acid, if there is any and again dried $24 \mathrm{~h}$ in atmosphere and $24 \mathrm{~h}$ in vacuum dessicator. The composites so obtained were then separated for further characterization and studies. The yield of the prepared composites was almost 1.96-1.98 g in each composition. Figure 1 shows the prepared composites.

\section{Characterization}

Prepared polymer composite and polymer nanocomposites were characterized by FTIR, XRD, FESEM and EDX analysis. XRD and FESEM were used for morphological characterization of nano phase while FTIR and EDX analysis were adopted for characterization of chemical moieties.

FTIR spectra of the samples were obtained using Attenuated Total Reflection (ATR) technique through Spectrum Two 95,163 (Perkin Elmer) FTIR spectrophotometer in $4000-400 \mathrm{~cm}^{-1}$ frequency range, at $2 \mathrm{~cm}^{-1} \mathrm{~s}^{-1}$ scanning rate.

The XRD patterns of polymer blend and their nanocomposites were studied using $\mathrm{Cu} \mathrm{K} \alpha$ radiation (1.54 $\AA$ A) through X'Pert Powder, PAN Analytical X-ray diffractometer. FESEM and EDX observations were performed through FESEM NOVA NANO 450 (FEI, Netherlands) model Scanning Electron Microscope. The sample to be characterized was placed on a specimen stub and after that platinum was plated on the upper surface of the sample to make it conductive.

\section{Application of developed polymer composite and polymer nanocomposites in removal of pollutants from water}

\section{Cleaning and pre-treatment of the composites before testing}

Preceding first use, the composites were cleaned to remove its preservatives. This cleaning procedure included following steps:

1. Rinsing with DM water.

2. Keeping in DM water and changing the water with certain intervals.

3. Rinsing with DM water again.

4. Filtering and drying of the composite.

The total dissolved solids (TDS) of DM water in permeate were measured using a TDS meter at periodic intervals during the steps. The procedure was continued until the TDS of the DM water in permeates became constant at 0 . 


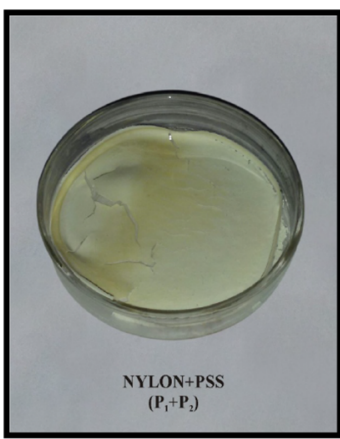

a

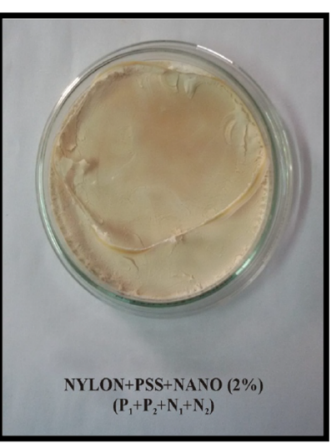

b

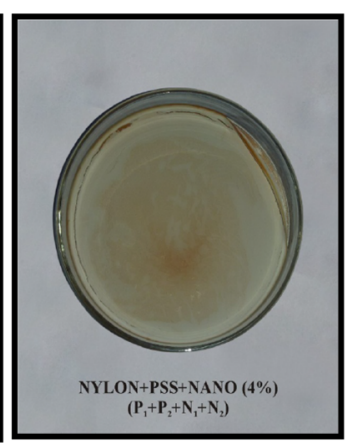

c

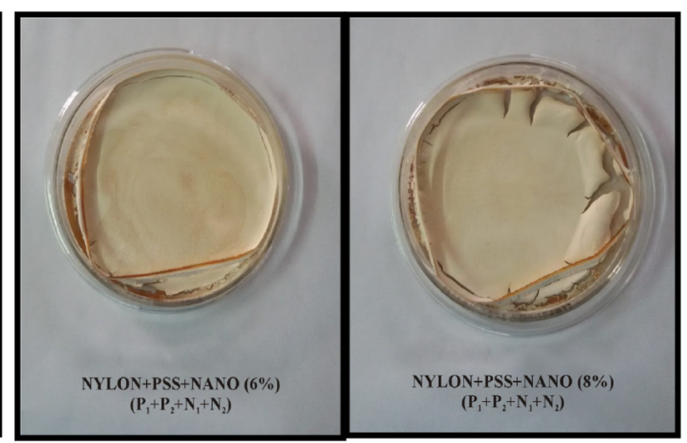

d

e

Fig. 1 Prepared samples of a polymer composite, b $2 \%$, c $4 \%$, d $6 \%$, and e $8 \%$ nanocomposites

\section{Preparation of standard solution}

A standard solution was prepared to analyze the removal capacity of prepared polymer and polymer nanocomposites. For this, $0.5 \mathrm{~g} \mathrm{CaCO}_{3}, 0.01 \mathrm{~g} \mathrm{NaF}^{0} 0.1 \mathrm{~g} \mathrm{KNO}_{3}$ and $0.1 \mathrm{~g} \mathrm{MgCl}_{2}$ were dissolved in distilled water and solution was made up to $1000 \mathrm{~mL}$.

\section{Removal of pollutants from polymer composite and polymer nanocomposites}

For studying the removal capacity of composites, a column of $30 \mathrm{~cm}$ height and $2 \mathrm{~cm}$ diameter (Fig. 2) was set in which layers of sterilized cotton, $1 \mathrm{~g}$ prepared composite and again cotton were placed and pressed to remove air completely. From this column, first DM water was passed and TDS of permeate was checked. The process was continued till the TDS of permeate became constant (zero) and $\mathrm{pH}$ was also constant during the whole process.

Then, 1 liter of standard solution was passed through the set column of polymer composite with maximum flow rate of 18 drops per minute and collected in pre-cleaned and rinsed bottle. The process was repeated with all composites-2, 4, $6,6 \%+\mathrm{Al}_{2} \mathrm{O}_{3}$ and $8 \%$ nanocomposites. With $6 \%$ nanocomposite, a layer of activated alumina $\left(\mathrm{Al}_{2} \mathrm{O}_{3}\right)$ was also placed on the layer of cotton followed by a layer of cotton and was pressed. Following that, to study the extent of removal of pollutants from composites, the standard solution and treated solutions (permeates) of composites were analyzed for the physico-chemical parameters.

\section{Results and discussion}

\section{Characterization}

\section{Fourier transform-infra red (FTIR) spectroscopy} measurement

To verify the bond formation between the polymers or between the alumina and iron oxide nanoparticles and the

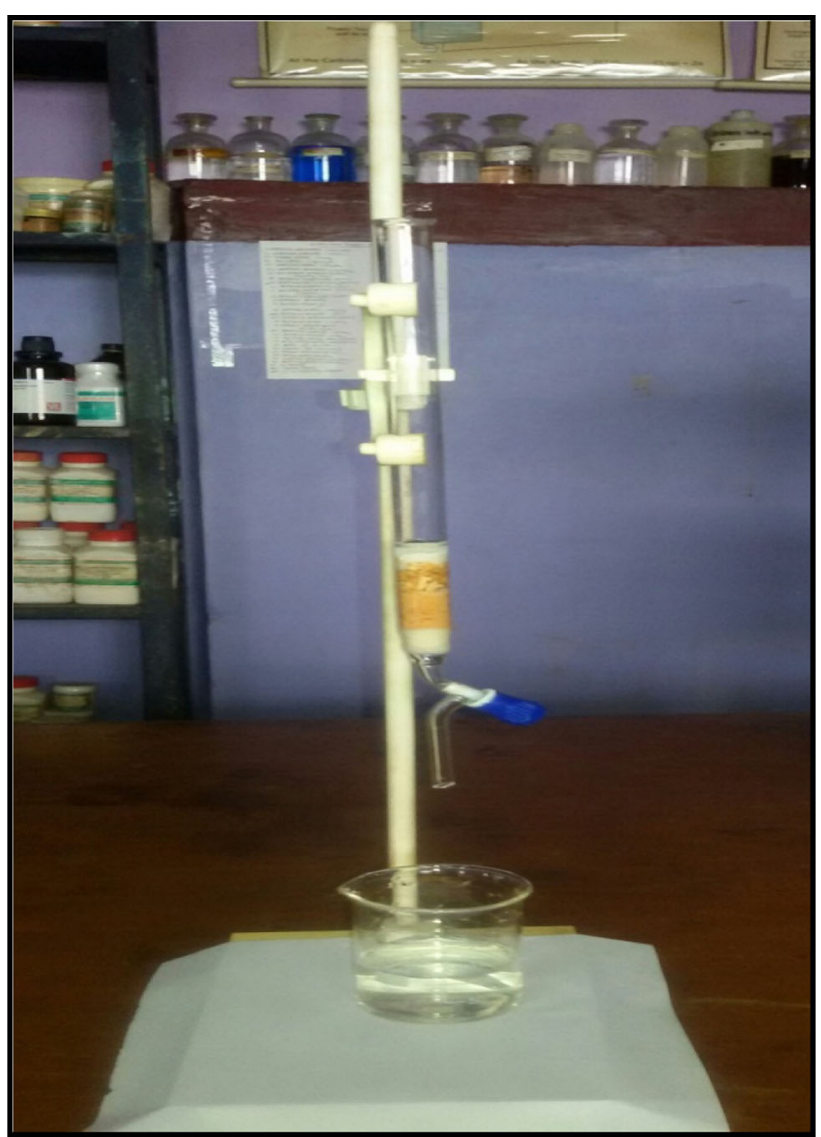

Fig. 2 Column used for the removal of pollutants from composite

polymers in prepared composites, FTIR spectrum was recorded for Nylon 6, 6, PSS, polymer composite, and 2, 4, $6,8 \%$ nanocomposites. The graphs are shown in Figs. 3, $4,5,6,7$, and 8 .

In Fig. 3, the peak in the range of $1680-1630$ is characteristic for $\mathrm{C}=\mathrm{O}$ stretch of amide and the peak in range $3500-3300$ is attributed to $\mathrm{N}-\mathrm{H}$ stretch of $2^{\circ}$ amides while in Fig. 4 the peak in the range of $1245-1155$ is representing $\mathrm{S}=\mathrm{O}$ stretch of sulphonate, and the peak in the range of 1080-1040 depicts the $\mathrm{SO}_{3}$ symmetric stretch of 
sulphonate group. Besides, these spectra of Nylon 6, 6 and PSS (Figs. 3 and 4) are compared with the standard graphs of the compounds given by Sigma-Aldrich, US from where these are procured and found out that the obtained spectra are the same as the standards.

The spectra of polymer composite, Fig. 5, and their nanocomposites spectra, Figs. 6, 7, 8, 9, exhibit the same characteristic peaks of Nylon 6, 6 and PSS as mentioned above. There is no appreciable appearance and disappearance of any extra peak. The spectra show that there is no bond formation between the two polymers and between the alumina and iron oxide nanoparticles. The polymer composite is confirmed by the absence of peaks due to bonding of polymer-polymer, $\mathrm{Al}_{2} \mathrm{O}_{3}$-polymer composite and $\mathrm{Fe}_{2} \mathrm{O}_{3}$-polymer composite.

\section{$X$-ray diffraction analysis}

$\mathrm{X}$-ray scattering analysis is primarily concerned with investigating the presence and size of nanoparticles. Figures 10 and 11 exhibit the XRD pattern of the polymers Nylon 6, 6 and PSS. The presence of broad peaks in Fig. 13 confirms the nano dimension of the particles in the composites. Figures 12 and 13 represent the XRD patterns of polymer composite and polymer nanocomposite. XRD pattern of polymer composite shows a broad hallow in the $2 \theta$ range of $10^{\circ}-35^{\circ}$, whereas the pattern of the respective nanocomposite suggests that the systems exhibit semi-crystalline structure. The presence of extra and sharp peaks in the XRD pattern of nanocomposite (in comparison with the XRD pattern of polymer composite) confirms the presence of $\mathrm{Al}_{2} \mathrm{O}_{3}$ and $\mathrm{Fe}_{2} \mathrm{O}_{3}$ nanoparticles in the composites. The peaks of the pattern were also compared with standard patterns given by analysis centre (GPCDS files).

\section{Field emission scanning electron microscopy (FESEM) and electron dispersive $X$-ray (EDX) analysis}

FESEM images were taken on the composite samples to examine the morphology of the composites formed, to analyze the distribution of nanoparticles, and to investigate the changes according to the change in concentration of the nanoparticles.

Figures 14, 15, 16, 17, and 18 show the morphology of the composites. The images suggest that nanoparticles are almost uniformly distributed in the polymer matrix. As expected by the used weight percentage of nanoparticles, the number of particles detected is very less in $2 \%$ composite (very rare to find out) and it successively increases with increasing the percentage of particles. The uniformity of particles is also more pronounced for higher concentration composite as seen from the images.

The particles are polydispersed, and some of them form multiparticle aggregates. FESEM images also showed some level of flocculation of nanoparticles, but of varying sizes that is why the size of nanoparticles varied from $35-230 \mathrm{~nm}$.

To identify and confirm the presence and composition of alumina and iron oxide nanoparticles in the composite, EDX measurement was also taken on the nanocomposite. The EDX graph of composite is shown in Fig. 19.

\section{Removal of pollutants from polymer composite and polymer nanocomposites}

To analyze the removal of pollutants from polymer composite and polymer nanocomposites standard solution and treated solutions of polymer composite $\left(\mathrm{P}_{1}+\mathrm{P}_{2}\right), 2,4,6$, $6+\mathrm{Al}_{2} \mathrm{O}_{3}, 8 \%$ nanocomposites were analyzed for physico-chemical parameters. The results are depicted in Table 1.

$p H \mathrm{pH}$ value reduced from 7.3 to 7.0. Reduction in the $\mathrm{pH}$ value by polymer composite is $1.37 \%$, while all other composites reduced the $\mathrm{pH}$ level to the same $4.1 \%$ extent.

Total alkalinity Total alkalinity reduced from 180 to $60 \mathrm{mg} / \mathrm{L}$. Polymer composite and $2 \%$ nanocomposite reduced the value almost up to the same 31.12 and $32.22 \%$ extent, respectively. Removal by $4 \%$ nanocomposite is
Fig. 3 FTIR spectra of nylon 6, 6

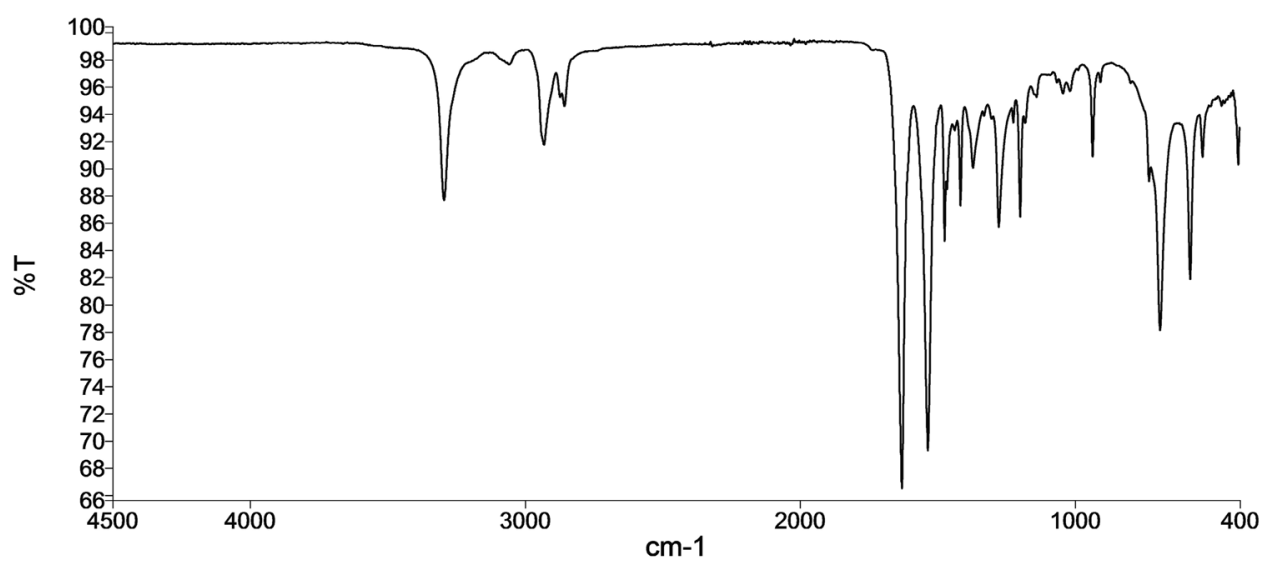


Fig. 4 FTIR spectra of PSS

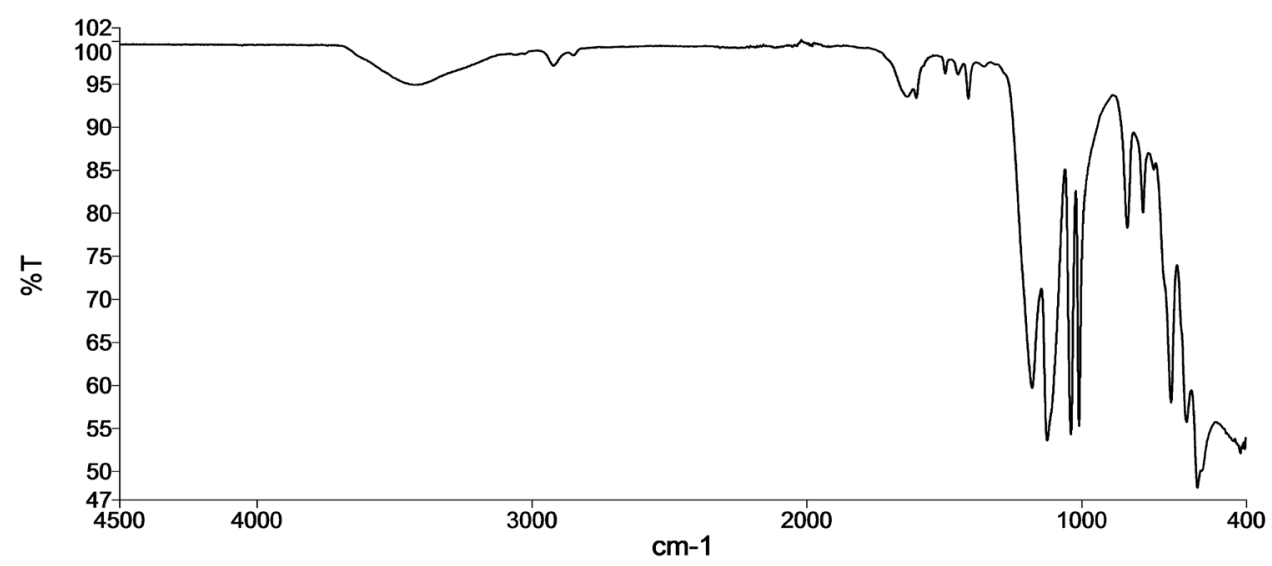

Fig. 5 FTIR spectra of polymer composite $\left(\mathrm{P}_{1}+\mathrm{P}_{2}\right)$
Fig. 6 FTIR spectra of $2 \%$ nanocomposite
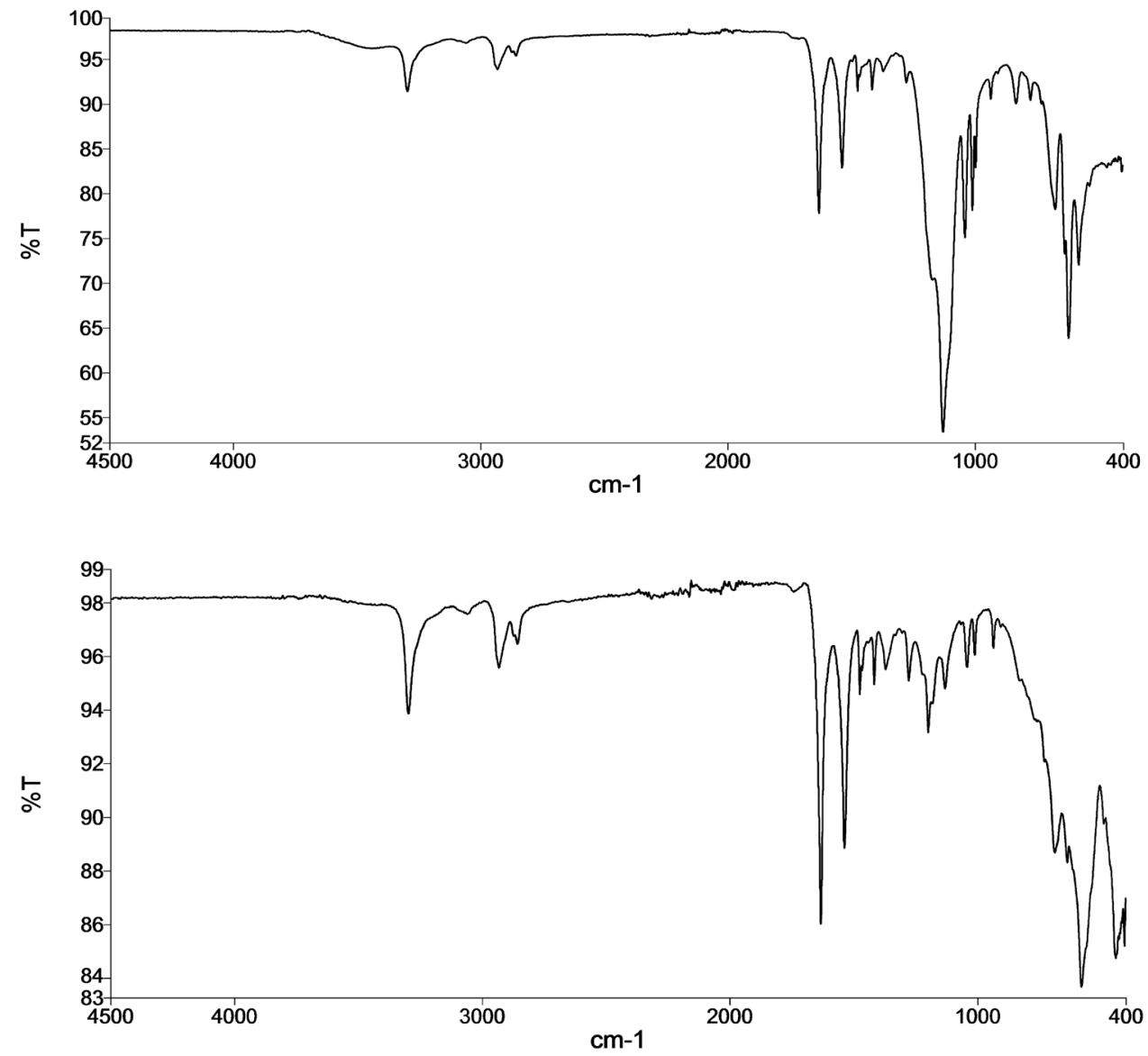

$63.89 \%$ and the highest removal is obtained from $6 \%$, $6 \%+\mathrm{Al}_{2} \mathrm{O}_{3}$ and $8 \%$ composites which is about $66.67 \%$.

Total hardness The values of total hardness reduced from 420 to $240 \mathrm{mg} / \mathrm{L}$. Removal of hardness is maximum and same $42.85 \%$ by polymer $\left(\mathrm{P}_{1}+\mathrm{P}_{2}\right), 2,4$, and $6 \%$ composites. Basically, removal of hardness is due to the presence of PSS polymer in the composite and that is why the removal percentage is not increasing with the increasing amount of nanocompounds. In permeates of $6 \%+\mathrm{Al}_{2} \mathrm{O}_{3}$ and $8 \%$ composites, value of hardness increases, and removal by these is only 15.71 and $16.66 \%$, respectively.

Calcium Removal of calcium follows the same trend as in total hardness. The maximum removal of calcium is $66.67 \%$ by polymer $\left(\mathrm{P}_{1}+\mathrm{P}_{2}\right), 2,4$, and $6 \%$ composites and minimum is $33.34 \%$ by $6 \%+\mathrm{Al}_{2} \mathrm{O}_{3}$ and $8 \%$ composites.

Magnesium Magnesium removal also exhibits the same trend as in total hardness and calcium with the highest value $25 \%$ reduction with polymer $\left(\mathrm{P}_{1}+\mathrm{P}_{2}\right), 2,4$, and 
Fig. 7 FTIR spectra of $4 \%$ nanocomposite

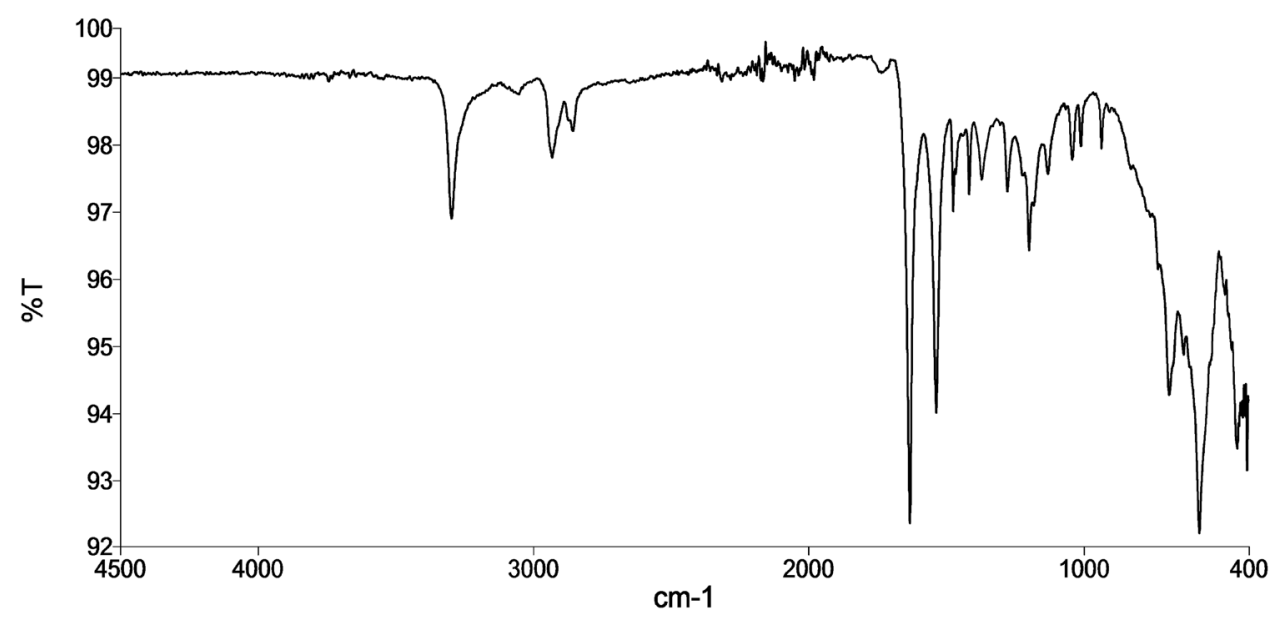

Fig. 8 FTIR spectra of $6 \%$ nanocomposite

Fig. 9 FTIR spectra of $8 \%$ nanocomposite

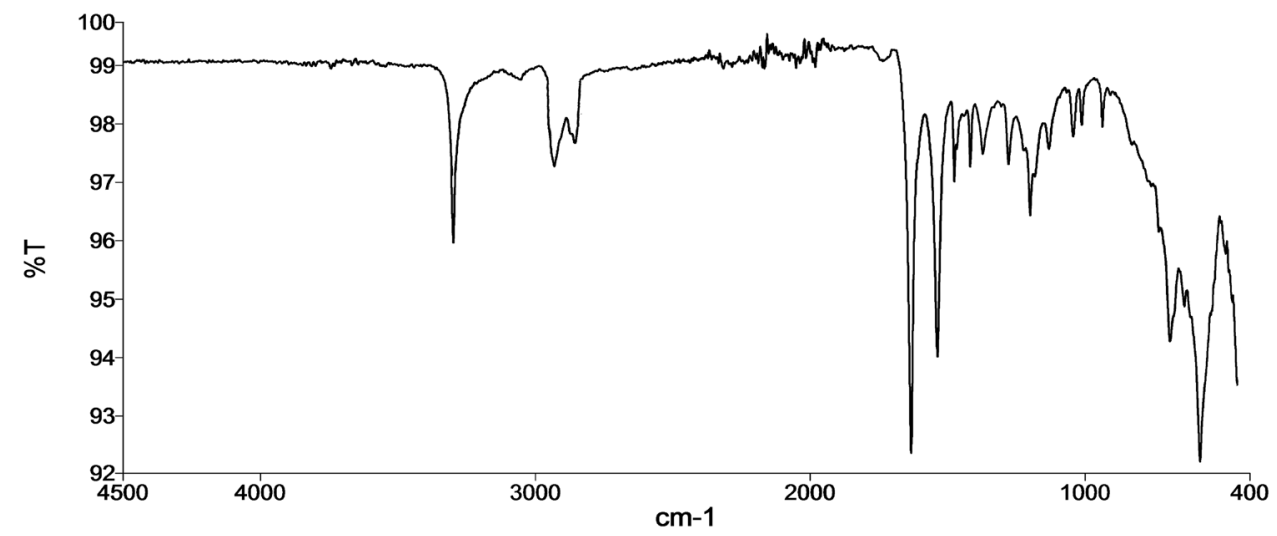

$6 \%$ composites and the lowest $2.5 \%$ with $6 \%+\mathrm{Al}_{2} \mathrm{O}_{3}$ composite. These values also depict the less removal of magnesium in comparison of calcium.

Chloride The maximum reduction found in chloride levels is $58.66 \%$ by polymer $\left(\mathrm{P}_{1}+\mathrm{P}_{2}\right), 2,4,6$ and $6 \%+\mathrm{Al}_{2} \mathrm{O}_{3}$ composites, by $8 \%$ composite removal was only $37 \%$. Consequently, it can be said that the removal of chloride is mainly due to the polymer matrix and in the composite of maximum percentage of nanocompounds the tendency of chloride removal decreases.

Nitrate The values of nitrate are reduced from 92 to $60 \mathrm{mg} / \mathrm{L}$. The maximum nitrate removal $(34.78 \%)$ is obtained from 6 and $6 \%+\mathrm{Al}_{2} \mathrm{O}_{3}$ composites. Polymer and $2 \%$ nanocomposite remove nitrate to the same extent $8.69 \%$ which is increased to a level of $21.73 \%$ by $4 \%$ composite, but in $8 \%$ composite removal percentage 
Fig. 10 XRD pattern of nylon 6,6

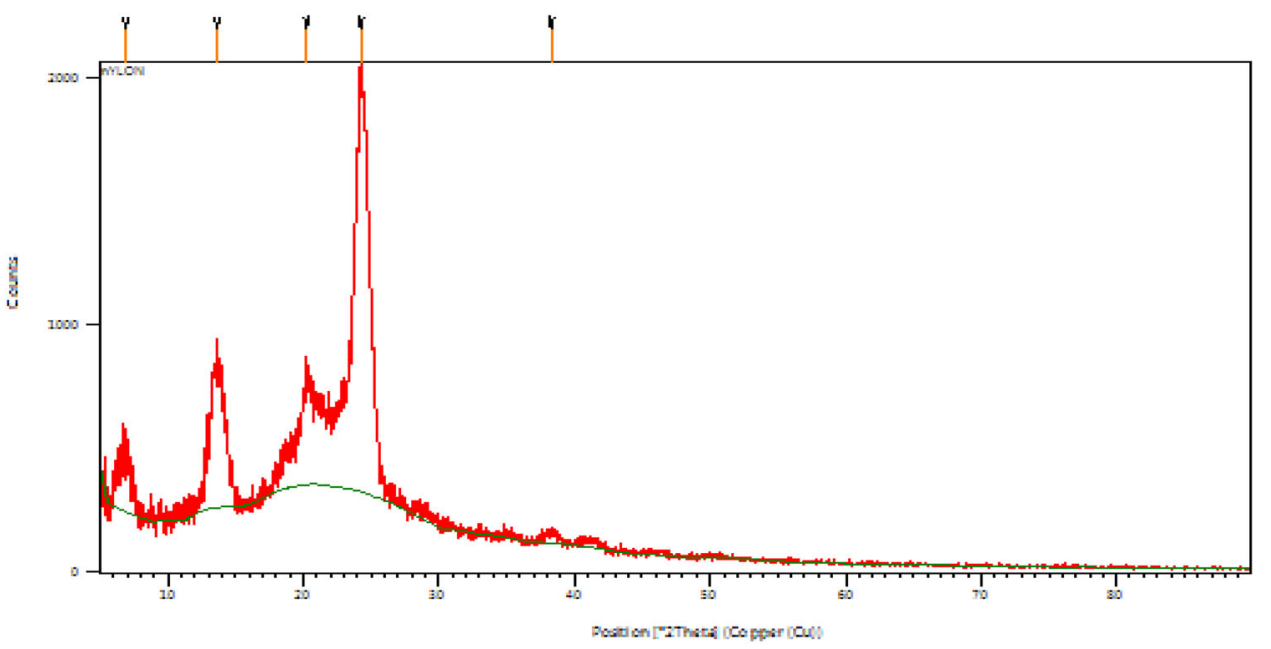

Fig. 11 XRD pattern of PSS

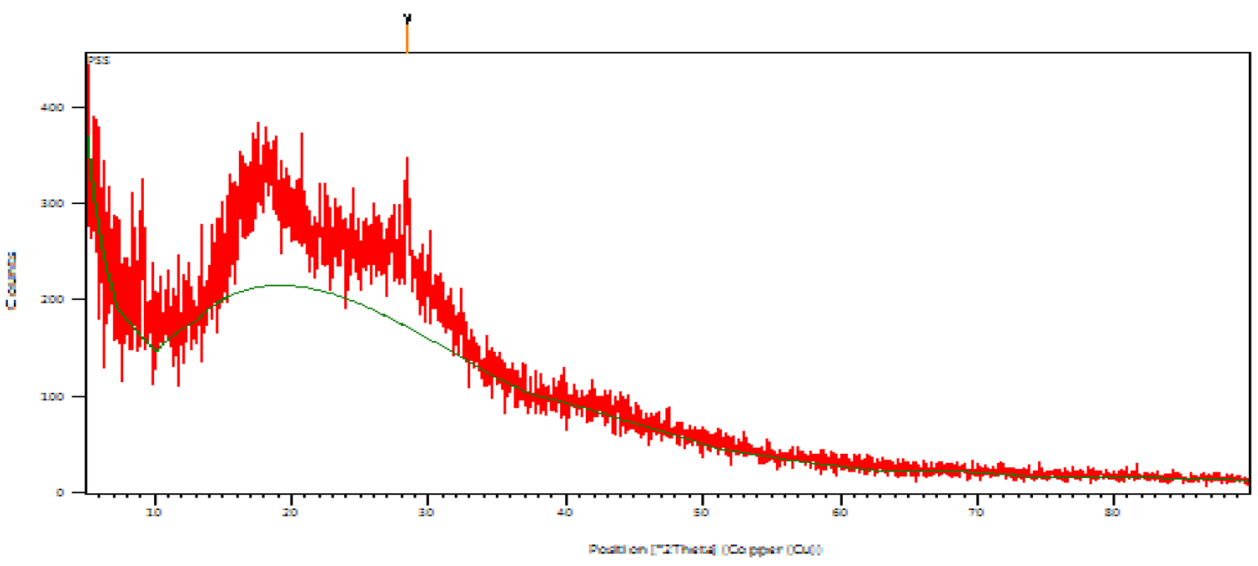

Fig. 12 XRD pattern of polymer composite

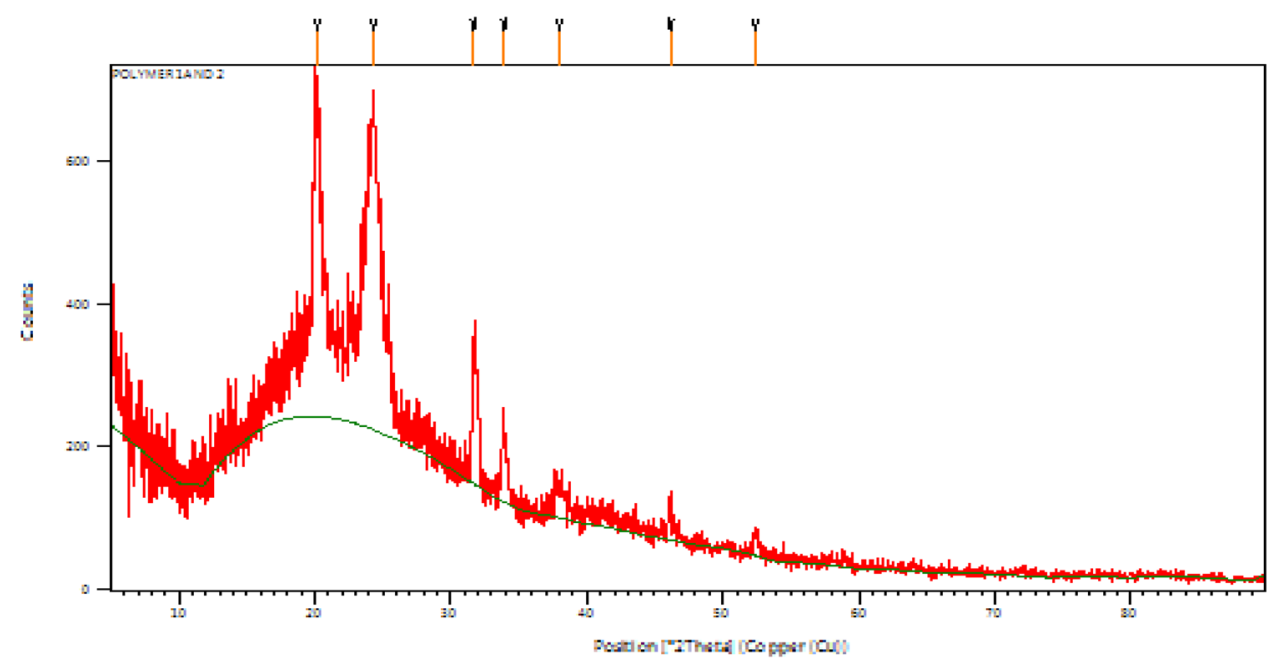

decreases to $9.78 \%$, showing the decreased removal capacity of the composite.

Fluoride From the results, it is observed that as the concentration of nanocompounds in the composites increases, the removal of fluoride by these composites also increases, but after attaining the maximum value removal tendency decreases in $8 \%$ nanocomposite. Percentages of the decrease are $39.75,45.78,51.80,63.85$, and $57.83 \%$ by polymer $\left(\mathrm{P}_{1}+\mathrm{P}_{2}\right), 2,4,6$, and $8 \%$ composites, respectively. Among these composites, maximum removal 
Fig. 13 XRD pattern of polymer nanocomposite

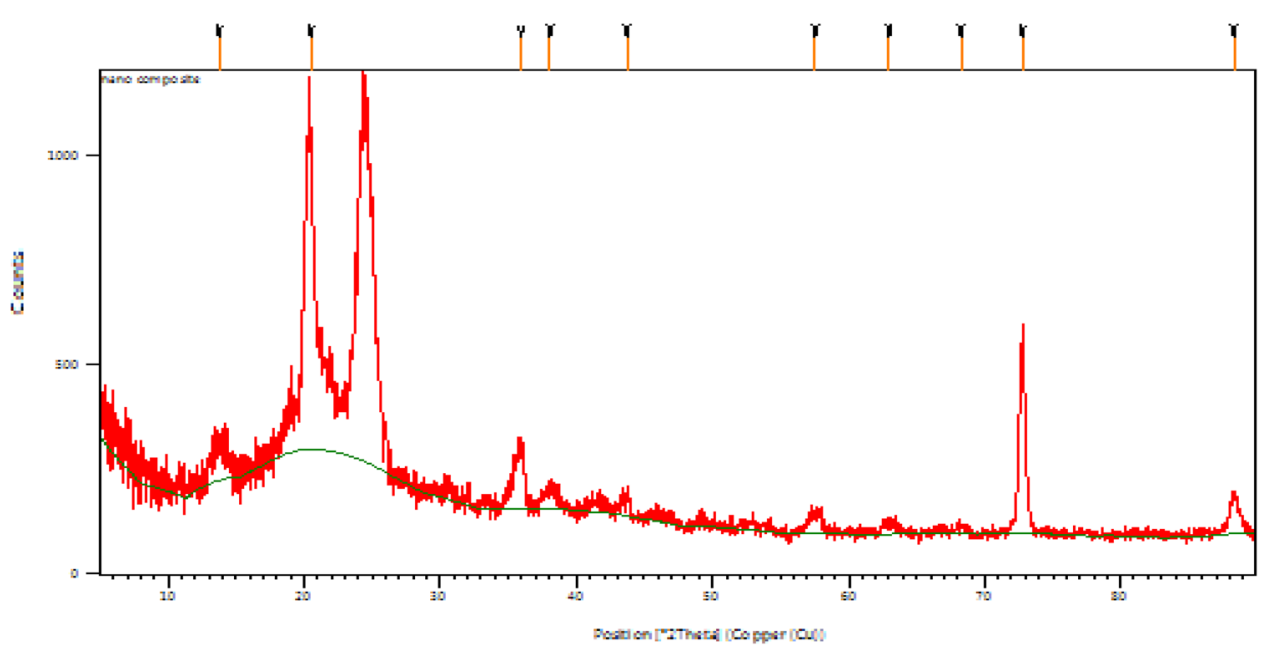

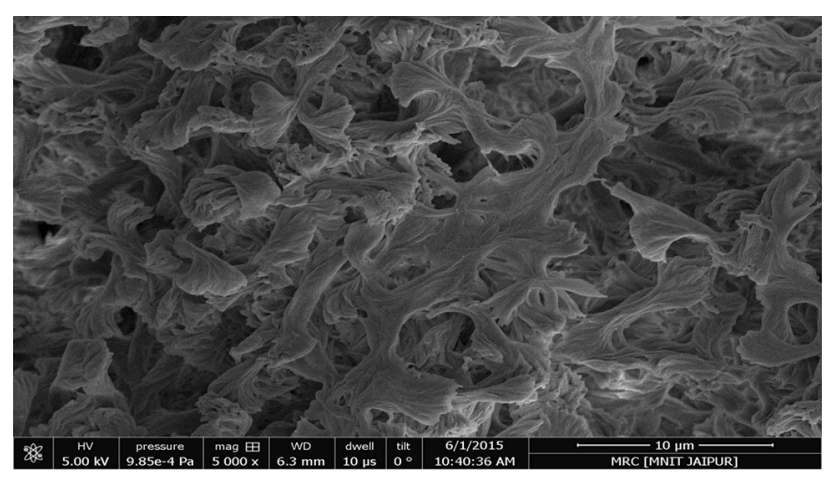

Fig. 14 SEM micrographs showing the surface morphology of polymer composite

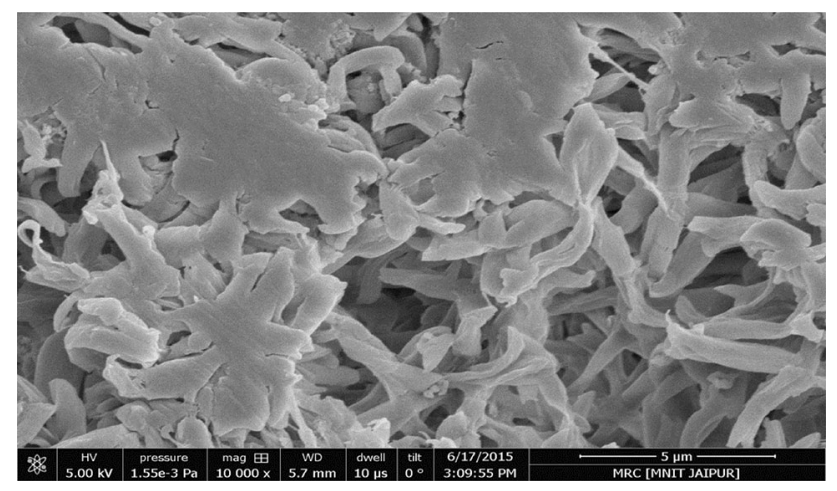

Fig. 15 SEM micrographs showing the surface morphology of $2 \%$ nanocomposite

$(63.85 \%)$ is obtained from $6 \%$ nanocomposite. When we placed a layer of activated alumina with this $6 \%$ nanocomposite in the column, the best results were obtained and fluoride removal reached a maximum of $93.97 \%$.

Total dissolved solids Values of TDS decreased from 470 to $276 \mathrm{mg} / \mathrm{L}$ exhibiting the same tendency as in fluoride reduction, but exceptionally TDS level is increased in

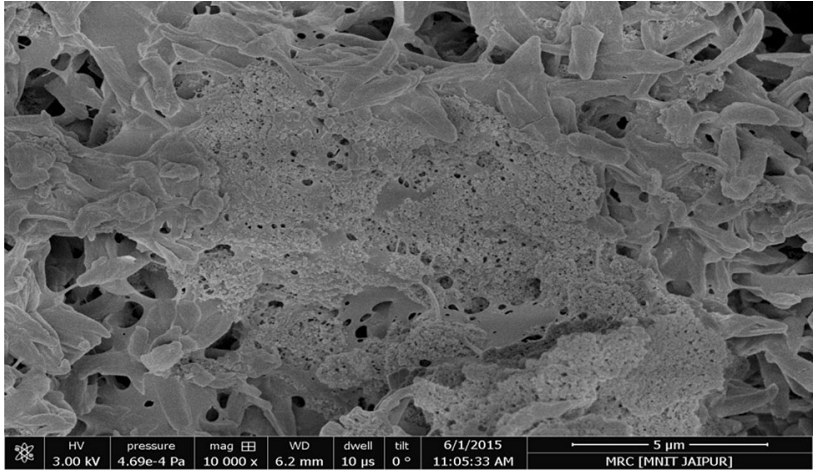

Fig. 16 SEM micrographs showing the surface morphology of $4 \%$ nanocomposite

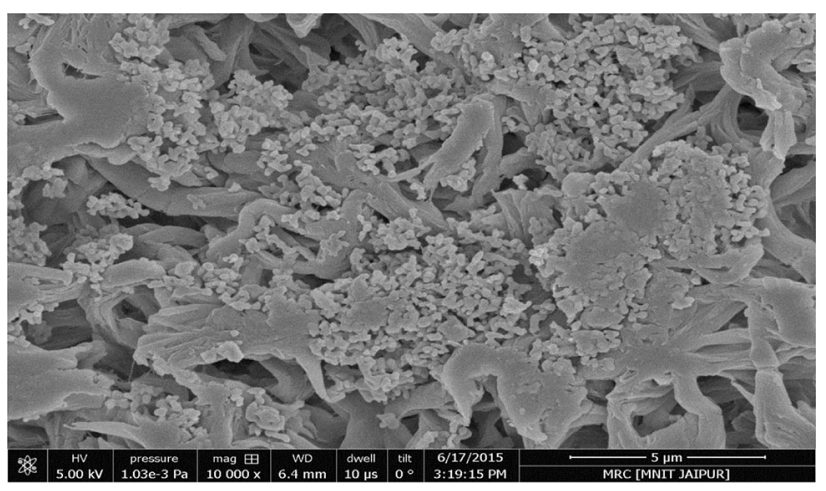

Fig. 17 SEM micrographs showing the surface morphology of $6 \%$ nanocomposite

treated solution of $6 \%+\mathrm{Al}_{2} \mathrm{O}_{3}$ composite in comparison to $6 \%$ composite. The obtained percentages decrease by polymer $\left(\mathrm{P}_{1}+\mathrm{P}_{2}\right), 2,4,6,6+\mathrm{Al}_{2} \mathrm{O}_{3}$ and $8 \%$ composites are, respectively, as follows: $15.53,24.68,26.17,41.27$, 28.72, and $37.02 \%$.

Electrical conductivity As EC is directly proportional to TDS, the trends for the reduction in the values of EC are the same as TDS. The best results are obtained from $6 \%$ 


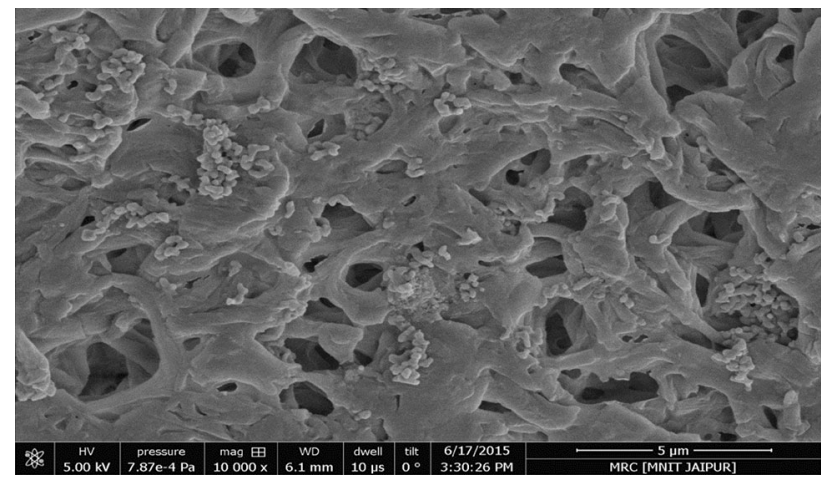

Fig. 18 SEM micrographs showing the surface morphology of $8 \%$ nanocomposite

composite $(41.37 \%)$ and the worst from polymer composite $(15.62 \%)$.

In this research, it was attempted to develop a cost effective, multifunctional composite for water treatment. The appropriate chemicals were chosen for preparation of composites. Since $6 \%$ nanocomposite provided the best results, the approximate cost of this composite was calculated and it was found that the treatment of one liter of water by $1 \mathrm{~g}$ of this composite costs almost 10.20 Indian rupees. This cost can also be reduced using commercial grade chemicals as for research purpose; the chemicals were of AR grade. It must be mentioned that this cost includes only the costs of chemicals and reagents used and does not contain the cost of apparatus design in which the composite would be used. The operating cost of apparatus is not also taken into account. But, due to its multifunctional ability and its cost in comparison to other developed techniques, it considerably assures the achievement of the purpose.
As mentioned, the developed composite has significant pollutant removal abilities, but it can be utilized at large scale or at commercial level after some more research regarding its practical aspects which mainly includes the regeneration process of the exhausted composite or the disposal of composite, measurement of maximum amount of water to be purified by certain amount of composites and particular retention time of nanoparticles in composite.

\section{Conclusions}

In the study, $6 \%$ nanocomposite provides the best results. It exhibits the maximum removal of all parameters among all composites. The removal of total alkalinity is $66.67 \%$, total hardness $42.85 \%$, calcium $66.67 \%$, magnesium $25 \%$, chloride $58.66 \%$, nitrate $34.78 \%$, fluoride $63.85 \%$, TDS $41.27 \%$, and EC is up to the level of $41.37 \%$ by this composite. Incorporation of a layer of activated alumina in the column with $6 \%$ composite increases the fluoride reduction from 63.85 to $93.97 \%$, but it increases the values of total hardness, calcium, magnesium, TDS and EC in treated solution (permeate), i.e., reduces the removal capacities for these parameters.

A particular trend is observed in removal of Nitrate, Fluoride, TDS and EC parameters. As the percentage of nanocompounds in the polymer increases, the removal of these pollutants also increases and reaches a maximum for $6 \%$ composite. But, by $8 \%$ composite removal of these pollutants decreases, i.e., depicts the reduced pollutant removal capacity of $8 \%$ composite. The results of total hardness, calcium and magnesium indicate that hardness removal is mainly due to the presence of PSS (polymer) in the composite, and the values of chloride removal suggest
Fig. 19 EDX graph showing the composition of nanocomposite

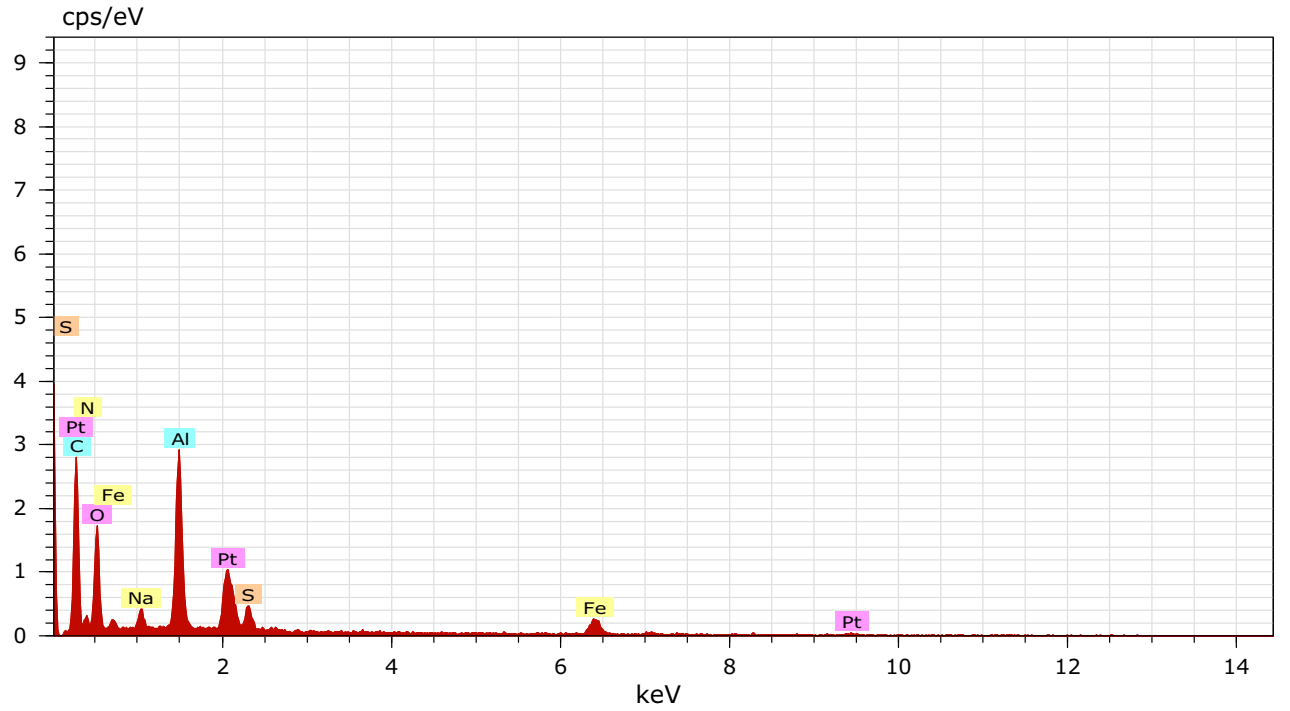




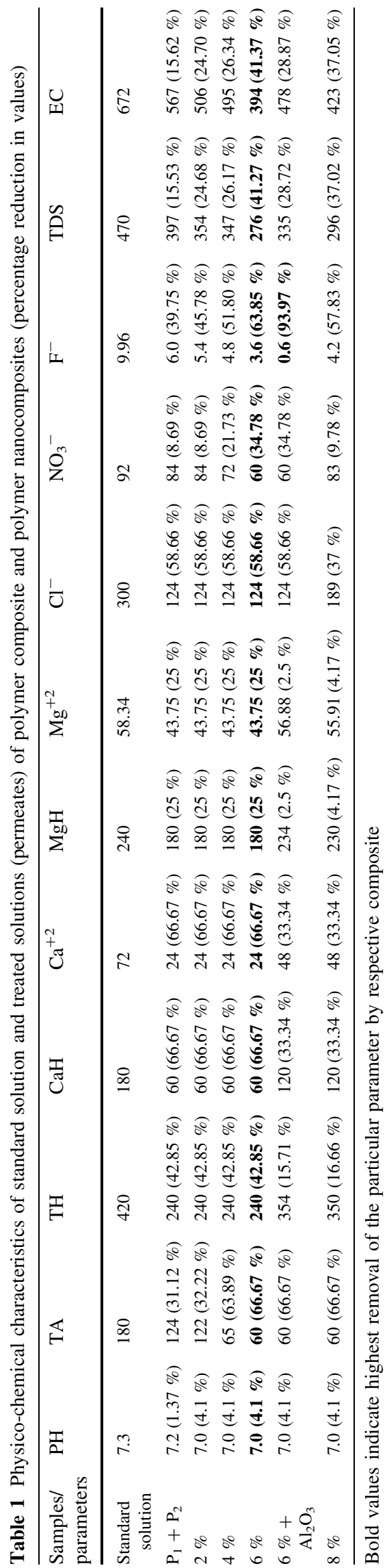

that its reduction is mainly by polymer matrix. Removal capacities for these parameters are not much affected or not affected at all by the percentage of nanocompound in the composite.

The study is a step towards developing multifunctional, cost-effective polymer nanocomposites for water remediation applications.

Author's contributions SS studied the previously prepared polymer metal nanocomposites and their uses in treatment of water, then selected the appropriate polymers and nanoparticles for the preparation of polymer composite and polymer metal nanocomposites. She prepared composites by suitable methods in the laboratory, studied the new characterization methods of composites, and interpreted their characterization analysis done in Material Research Center, MNIT, Jaipur. After that, she analyzed the pollutant removal capacities of prepared composites by setting a column and tested treated solutions (permeates) of composites for certain physico-chemical parameters of water. Finally, she interpreted the results and drafted the manuscript. US guided the SS in initial studies of the previously prepared polymer metal nanocomposites and their uses in treatment of water and finalized the appropriate polymers and nanoparticles for the preparation of composites; provided the laboratory facility for preparation of composites, study of pollutant removal capacities of composites and for the analysis of physico-chemical parameters of water samples (standard solution and permeates); supervised the whole work critically and keenly; and helped in interpretation of the results, draft of manuscript and approved the final version of paper.

Acknowledgments One of the authors is grateful to Mrs. Ritu Saxena, Director, Chitransh Public Sr. Sec. School, Jaipur, Rajasthan for her valuable guidance and abundant succor in this research work. Author also acknowledges the support of Material Research Centre, Malviya National Institute of Technology, Jaipur, Rajasthan in characterization analysis.

Open Access This article is distributed under the terms of the Creative Commons Attribution 4.0 International License (http://creative commons.org/licenses/by/4.0/), which permits unrestricted use, distribution, and reproduction in any medium, provided you give appropriate credit to the original author(s) and the source, provide a link to the Creative Commons license, and indicate if changes were made.

\section{References}

1. Progress on Sanitation and Drinking-Water: World Health Organization (WHO)/United Nations International Children's Fund (UNICEF). Joint Monitoring Programme for Water Supply and Sanitation, (2010)

2. Coping with water scarcity-Challenge of the twenty-first century: United Nations (UN) Water. Food and Agricultural Association (FAO), (2007) 
3. Sundara, K.P., Ratnakanth, M.J., Rao, H.: Assessment and mapping of groundwater quality using geographical information systems. Int. J. Eng. Sci. Tech. 2(11), 6035-6046 (2010)

4. Trivedi, P.R.: India's environment. APH Publishing Corporation, New Delhi (2004)

5. Balamurugan, R., Sundarrajan, S., Ramakrishna, S.: Recent trends in nanofibrous membranes and their suitability for air and water filtrations. Mem. 1, 232-248 (2011)

6. Rout, T.K., Verma, R., Dennis, R.V., Banerjee, S.: Study the removal of fluoride from aqueous medium by using nano-composites. J. Encap. Adso. Sci. 5, 38-52 (2015)

7. Zhou, H., Chen, W., Zhang, X., Shi, D.: Preparation of nanometer magnesia and its properties for fluoride removal. Fron. Env. Sci. 3(3), 97-108 (2014)

8. Tomar, V., Kumar, D.: A critical study on efficiency of different materials for fluoride removal from aqueous media. Che. Cen. J. 7(51), 1-15 (2013)

9. Kumar, E.: Removal of inorganic anionic pollutants from water using adsorption technology. Linnaeus University Dissertations. Linnaeus University Press, Växjö (2013)

10. Kumar, V., Talreja, N., Deva, D., Sankararamakrishnan, N., Sharma, A., Verma, N.: Development of bi-metal doped microand nano multi-functional polymeric adsorbents for the removal of fluoride and arsenic (V) from wastewater. Desal. 282, 27-38 (2011)

11. Ramos, R.L., Castillo, N.A., Azuara, A.J., Barron, J.M., Rodriguez, L.E.L., Rosales, J.M.M., Pina, A.A.: Fluoride removal from water solution by adsorption on activated alumina prepared from pseudo-boehmite. J. Env. Eng. Man. 18(5), 301-309 (2008)

12. Manimegalai, G., Shanthakumar, S., Sharma, C.: Pesticide mineralization in water using silver nanoparticles. Int. J. Chem. Sci. 9(3), 1463-1471 (2011)

13. Manimegalai, G., Shanthakumar, S., Sharma, C.: Silver nanoparticles: synthesis and application in mineralization of pesticides using membrane support. Int. Nano. Lett. 4, 105 (2014). doi:10.1007/s40089-014-0105-8

14. Saifuddin, N., Nian, C.Y., Zhan, L.W., Ning, K.X.: Chitosansilver nanoparticles composite as point-of-use drinking water filtration system for household to remove pesticides in water. Asian J. Biochem. 6(2), 142-159 (2011). doi:10.3923/ajb.2011. 142.159

15. Ratpukdi, S.S., Furhacker, M.: Review: issues of silver nanoparticles in engineered environment treatment systems. Water Air. Soil. Pollut. 225, 1939 (2014). doi:10.1007/s11270014-1939-4

16. Azari, A., Mahvi, A.H., Naseri, S., Kalantary, R.R., Saberi, M.: Nitrate removal from aqueous solution by using modified clinoptilolite zeolite. Arch. Hyg. Sci. 3(1), 21-29 (2014)

17. Ahmad, H.B., Abbas, Y., Hussain, M., Akhtar, N., Ansari, T.M., Zuber, M., Zia, K.M., Arain, S.A.: Synthesis and application of alumina supported nano zero valent zinc as adsorbent for the removal of arsenic and nitrate. Kor. J. Che. Eng. 31(2), 284-288 (2014)

18. Mukherjee, R., De, S.: Adsorptive removal of nitrate from aqueous solution by polyacrylonitrile-alumina nanoparticle mixed matrix hollow-fiber membrane. J. Mem. Sci. 466, 281-292 (2014)

19. Katal, R., Pourkarimi, S., Bahmani, E., Dehkordi, H.A., Ghayyem, M.A., Esfandian, H.: Synthesis of $\mathrm{Fe}_{3} \mathrm{O}_{4} /$ Polyaniline nanocomposite and its application for nitrate removal from aqueous solutions. J. Vin. Add. Tech. 19, 147-156 (2013). doi:10. $1002 / \mathrm{vnl} .21306$

20. Nabid, M.R., Sedghi, R., Sharifi, R., Oskooie, H.A., Heravi, M.M.: Removal of toxic nitrate ions from drinking water using conducting polymer/MWCNTs nanocomposites. Iran. Pol. J. 22(2), 85-92 (2012). doi:10.1007/s13726-012-0106-2

21. Bhatnagar, A., Kumar, E., Sillanpa, M.: Nitrate removal from water by nano-alumina: characterization and sorption studies. Che. Eng. J. 163(3), 317-323 (2010)

22. Sedaghat, S., Nasseri, A.: Synthesis and stabilization of Ag nanoparticles on a polyamide (nylon 6, 6) surface and its antibacterial effects. Int. Nan. Lett. 1(1), 22-24 (2011)

23. Marek, J., Beneš, M.J., Jelínek, L.: Cation and anion exchangers from nanofibrous polystyrene for fast water treatment. Nan. Con. 20(22), 1-6 (2009) 